\title{
Local Fractional Variational Iteration Method for Inhomogeneous Helmholtz Equation within Local Fractional Derivative Operator
}

\author{
Xian-Jin Wang, ${ }^{1}$ Yang Zhao, ${ }^{2}$ Carlo Cattani, ${ }^{3}$ and Xiao-Jun Yang ${ }^{4}$ \\ ${ }^{1}$ Foundation College, Ningbo Dahongying University, Ningbo 315175, China \\ ${ }^{2}$ Electronic and Information Technology Department, Jiangmen Polytechnic, Jiangmen 529090, China \\ ${ }^{3}$ Department of Mathematics, University of Salerno, Via Giovanni Paolo II, Fisciano, 84084 Salerno, Italy \\ ${ }^{4}$ Department of Mathematics and Mechanics, China University of Mining and Technology, Xuzhou, Jiangsu 221008, China
}

Correspondence should be addressed to Yang Zhao; zhaoyang19781023@gmail.com

Received 27 May 2014; Accepted 6 June 2014; Published 26 June 2014

Academic Editor: Abdon Atangana

Copyright (C) 2014 Xian-Jin Wang et al. This is an open access article distributed under the Creative Commons Attribution License, which permits unrestricted use, distribution, and reproduction in any medium, provided the original work is properly cited.

\begin{abstract}
The inhomogeneous Helmholtz equation within the local fractional derivative operator conditions is investigated in this paper. The local fractional variational iteration method is applied to obtain the nondifferentiable solutions and the graphs of the illustrative examples are also shown.
\end{abstract}

\section{Introduction}

Helmholtz equation has played an important role in the partial differential equations arising in mathematical physics $[1,2]$. In computing the solution of Helmholtz equation, some analytical and numerical methods were presented. For example, Ihlenburg and Babuška used the finite element method to deal with the Helmholtz equation [3]. Momani and Abuasad suggested the variational iteration method to solve the Helmholtz equation [4]. Rafei and Ganji reported the homotopy perturbation method to report the solution to the Helmholtz equation [5]. Bayliss et al. considered the iterative method to discuss the Helmholtz equation [6]. Benamou and Desprès reported the domain decomposition method for solving the Helmholtz equation [7]. Linton presented Green's function method for the Helmholtz equation [8]. Singer and Turkel proposed the finite difference method to solve the Helmholtz equation [9]. Otto and Larsson applied the second-order method to discuss the Helmholtz equation [10].

Recently, the fractional calculus [11, 12] was developed and applied to present some models in the fields, such as the fractional-order digital control systems [13], the fractional-order viscoelasticity [14], the fractional-order quantum mechanics [15], and fractional-order dynamics [16]. More recently, Samuel and Thomas report the fractional Helmholtz equations [17] and some methods for solving the fractional differential equations were reported in [18-23]. However, we are faced with the problem that there must be some calculus to deal with the nondifferentiable solution for Helmholtz equation, which was structured within the local fractional derivative [24-34]. In this paper, we consider the local fractional inhomogeneous Helmholtz equation in twodimensional case [31, 32]:

$$
\frac{\partial^{2 \alpha} M(x, y)}{\partial x^{2 \alpha}}+\frac{\partial^{2 \alpha} M(x, y)}{\partial y^{2 \alpha}}+\omega^{2 \alpha} M(x, y)=f(x, y),
$$

where $f(x, y)$ is a local fractional continuous function and the local fractional partial derivative is defined as follows [24]:

$$
\left.\frac{\partial^{\alpha} f(x, y)}{\partial x^{\alpha}}\right|_{x=x_{0}}=\lim _{x \rightarrow x_{0}} \frac{\Delta^{\alpha}\left(f(x, y)-f\left(x_{0}, y\right)\right)}{\left(x-x_{0}\right)^{\alpha}},
$$


with

$$
\begin{aligned}
& \Delta^{\alpha}\left(f(x, y)-f\left(x_{0}, y\right)\right) \\
& \quad \cong \Gamma(1+\alpha) \Delta\left(f(x, y)-f\left(x_{0}, y\right)\right) .
\end{aligned}
$$

The local fractional inhomogeneous Helmholtz equation in three-dimensional case was suggested as follows [29, 30]:

$$
\begin{gathered}
\frac{\partial^{2 \alpha} M(x, y, z)}{\partial x^{2 \alpha}}+\frac{\partial^{2 \alpha} M(x, y, z)}{\partial y^{2 \alpha}}+\frac{\partial^{2 \alpha} M(x, y, z)}{\partial z^{2 \alpha}} \\
+\omega^{2 \alpha} M(x, y, z)=f(x, y, z),
\end{gathered}
$$

where $f(x, y, z)$ is a local fractional continuous function. Here, we use the local fractional variational iteration method [30-34] to solve the local fractional inhomogeneous Helmholtz equation in two-dimensional case. The structure of this paper is as follows. In Section 2, we analyze the local fractional variational iteration method. In Section 3, we present some illustrative examples. Finally, the conclusion is given in Section 4.

\section{Analysis of the Local Fractional Variational Iteration Method}

Here, we give the analysis of the local fractional variational iteration method as follows. We first consider the local fractional linear partial differential equation:

$$
L_{\alpha} u+R_{\alpha} u=g(t),
$$

where $L_{\alpha}$ denotes linear local fractional derivative operator of order $2 \alpha, R_{\alpha}$ denotes a lower-order local fractional derivative operator, and $g(t)$ is the nondifferentiable source term.

Let the local fractional operator be defined as [24, 30-34]

$$
\begin{aligned}
{ }_{a} I_{b}^{(\alpha)} f(x) & =\frac{1}{\Gamma(1+\alpha)} \int_{a}^{b} f(t)(d t)^{\alpha} \\
& =\frac{1}{\Gamma(1+\alpha)} \lim _{\Delta t \rightarrow 0} \sum_{j=0}^{j=N-1} f\left(t_{j}\right)\left(\Delta t_{j}\right)^{\alpha}
\end{aligned}
$$

with the partitions of the interval $[a, b], \Delta t_{j}=t_{j+1}-t_{j}, \Delta t=$ $\max \left\{\Delta t_{1}, \Delta t_{2}, \Delta t_{j}, \ldots\right\}$, and $j=0, \ldots, N-1, t_{0}=a, t_{N}=b$.

We now structure the correctional local fractional functional in the form

$$
\begin{aligned}
u_{n+1}(x)= & u_{n}(x)+{ }_{0} I_{x}^{(\alpha)} \\
& \times\left\{\zeta(s)\left(L_{\alpha} u_{n}(s)+R_{\alpha} u_{n}(s)-g(s)\right)\right\} .
\end{aligned}
$$

Making the local fractional variation, we have

$$
\begin{aligned}
\delta^{\alpha} u_{n+1}(x)= & \delta^{\alpha} u_{n}(x)+{ }_{0} I_{x}^{(\alpha)} \delta^{\alpha} \\
& \times\left\{\zeta(s)\left(L_{\alpha} u_{n}(s)+R_{\alpha} \widetilde{u}_{n}(s)-\tilde{g}(s)\right)\right\}=0
\end{aligned}
$$

such that the following stationary conditions are given as

$$
\begin{gathered}
1-\left.\zeta(s)^{(\alpha)}\right|_{s=x}=0,\left.\quad \zeta(s)\right|_{s=x}=0, \\
\left.\zeta(s)^{(2 \alpha)}\right|_{s=x}=0 .
\end{gathered}
$$

In view of (9), we obtain the fractal Lagrange multiplier, which is given by

$$
\zeta(s)=\frac{(s-x)^{\alpha}}{\Gamma(1+\alpha)} .
$$

From (7) and (10), we reach at the local fractional variational iteration algorithm

$$
\begin{aligned}
u_{n+1}(x)= & u_{n}(x)+{ }_{0} I_{x}^{(\alpha)} \\
& \times\left\{\frac{(s-x)^{\alpha}}{\Gamma(1+\alpha)}\left(L_{\alpha} u_{n}(s)+R_{\alpha} u_{n}(s)-g(s)\right)\right\},
\end{aligned}
$$

where the nondifferentiable initial value is suggested as

$$
u_{0}(x)=u(0)+\frac{x^{\alpha}}{\Gamma(1+\alpha)} u^{(\alpha)}(0) .
$$

Therefore, from (11), we write the solution of (7) as follows:

$$
u=\lim _{n \rightarrow \infty} u_{n}
$$

\section{Some Illustrative Examples}

In this section, we give some illustrative examples for solving the local fractional inhomogeneous Helmholtz equation in two-dimensional case.

We present the following local fractional inhomogeneous Helmholtz equation:

$$
\frac{\partial^{2 \alpha} M(x, y)}{\partial x^{2 \alpha}}+\frac{\partial^{2 \alpha} M(x, y)}{\partial y^{2 \alpha}}+M(x, y)=\frac{x^{\alpha}}{\Gamma(1+\alpha)} E_{\alpha}\left(y^{\alpha}\right),
$$

subject to the initial-boundary conditions:

$$
\begin{gathered}
\frac{\partial^{\alpha} M(0, y)}{\partial x^{\alpha}}=E_{\alpha}\left(-y^{\alpha}\right), \\
M(0, y)=0 .
\end{gathered}
$$

Making use of (11), we structure the local fractional variational iteration algorithm as follows:

$$
\begin{aligned}
M_{n+1}(x, y) & \\
= & M_{n}(x, y)+{ }_{0} I_{x}^{(\alpha)} \frac{(s-x)^{\alpha}}{\Gamma(1+\alpha)} \\
\quad \times & \left\{\frac{\partial^{2 \alpha} M_{n}(x, y)}{\partial x^{2 \alpha}}+\frac{\partial^{2 \alpha} M_{n}(x, y)}{\partial y^{2 \alpha}}\right. \\
& \left.+M_{n}(x, y)-\frac{x^{\alpha}}{\Gamma(1+\alpha)} E_{\alpha}\left(y^{\alpha}\right)\right\},
\end{aligned}
$$

where the initial value is presented as

$$
M_{0}(x, y)=\frac{x^{\alpha}}{\Gamma(1+\alpha)} E_{\alpha}\left(-y^{\alpha}\right),
$$

whose plot is shown in Figure 1. 


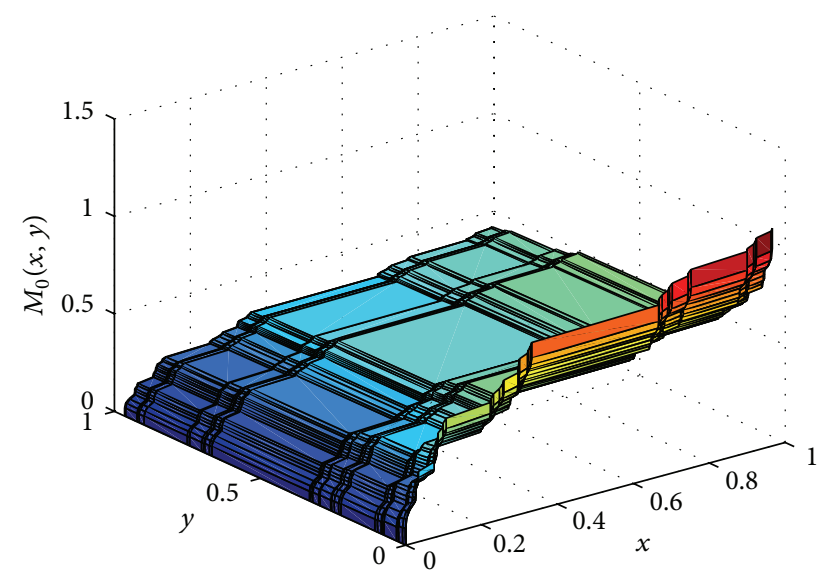

FIgURE 1: The graph of the initial value (17) where $\alpha=\ln 2 / \ln 3$.

In view of (16) and (17), we arrive at the first approximation:

$$
\begin{aligned}
M_{1}(x, y)= & M_{0}(x, y)+{ }_{0} I_{x}^{(\alpha)} \frac{(s-x)^{\alpha}}{\Gamma(1+\alpha)} \\
& \times\left\{\frac{\partial^{2 \alpha} M_{0}(x, y)}{\partial x^{2 \alpha}}+\frac{\partial^{2 \alpha} M_{0}(x, y)}{\partial y^{2 \alpha}}+M_{0}(x, y)\right. \\
& \left.-\frac{x^{\alpha}}{\Gamma(1+\alpha)} E_{\alpha}\left(-y^{\alpha}\right)\right\} \\
= & \frac{x^{\alpha}}{\Gamma(1+\alpha)} E_{\alpha}\left(-y^{\alpha}\right) \\
& +{ }_{0} I_{x}^{(\alpha)} \frac{(s-x)^{\alpha}}{\Gamma(1+\alpha)}\left\{\frac{x^{\alpha}}{\Gamma(1+\alpha)} E_{\alpha}\left(-y^{\alpha}\right)\right\} \\
= & \frac{x^{\alpha}}{\Gamma(1+\alpha)} E_{\alpha}\left(-y^{\alpha}\right)-\frac{x^{3 \alpha}}{\Gamma(1+3 \alpha)} E_{\alpha}\left(-y^{\alpha}\right) \\
= & \left(\frac{x^{\alpha}}{\Gamma(1+\alpha)}-\frac{x^{3 \alpha}}{\Gamma(1+3 \alpha)}\right) E_{\alpha}\left(-y^{\alpha}\right) .
\end{aligned}
$$

The second approximation is

$$
\begin{aligned}
M_{2}(x, y)= & M_{1}(x, y)+{ }_{0} I_{x}^{(\alpha)} \frac{(s-x)^{\alpha}}{\Gamma(1+\alpha)} \\
& \times\left\{\frac{\partial^{2 \alpha} M_{1}(x, y)}{\partial x^{2 \alpha}}+\frac{\partial^{2 \alpha} M_{1}(x, y)}{\partial y^{2 \alpha}}+M_{1}(x, y)\right. \\
& \left.-\frac{x^{\alpha}}{\Gamma(1+\alpha)} E_{\alpha}\left(-y^{\alpha}\right)\right\} \\
= & \left(\frac{x^{\alpha}}{\Gamma(1+\alpha)}-\frac{x^{3 \alpha}}{\Gamma(1+3 \alpha)}\right) E_{\alpha}\left(-y^{\alpha}\right) \\
& +{ }_{0} I_{x}^{(\alpha)} \frac{(s-x)^{\alpha}}{\Gamma(1+\alpha)}\left\{-\frac{2 x^{3 \alpha}}{\Gamma(1+3 \alpha)} E_{\alpha}\left(-y^{\alpha}\right)\right\}
\end{aligned}
$$

$$
\begin{aligned}
= & \frac{x^{\alpha}}{\Gamma(1+\alpha)} E_{\alpha}\left(-y^{\alpha}\right)-\frac{x^{3 \alpha}}{\Gamma(1+3 \alpha)} E_{\alpha}\left(-y^{\alpha}\right) \\
& +\frac{2 x^{5 \alpha}}{\Gamma(1+5 \alpha)} E_{\alpha}\left(-y^{\alpha}\right) \\
= & \left(\frac{x^{\alpha}}{\Gamma(1+\alpha)}-\frac{x^{3 \alpha}}{\Gamma(1+3 \alpha)}+\frac{2 x^{5 \alpha}}{\Gamma(1+5 \alpha)}\right) \\
& \times E_{\alpha}\left(-y^{\alpha}\right) .
\end{aligned}
$$

Making best of (16) and (19), the third approximation reads as

$$
\begin{aligned}
& M_{3}(x, y)=M_{2}(x, y)+{ }_{0} I_{x}^{(\alpha)} \frac{(s-x)^{\alpha}}{\Gamma(1+\alpha)} \\
& \times\left\{\frac{\partial^{2 \alpha} M_{2}(x, y)}{\partial x^{2 \alpha}}+\frac{\partial^{2 \alpha} M_{2}(x, y)}{\partial y^{2 \alpha}}+M_{2}(x, y)\right. \\
& \left.-\frac{x^{\alpha}}{\Gamma(1+\alpha)} E_{\alpha}\left(-y^{\alpha}\right)\right\} \\
& =\left(\frac{x^{\alpha}}{\Gamma(1+\alpha)}-\frac{x^{3 \alpha}}{\Gamma(1+3 \alpha)}+\frac{2 x^{5 \alpha}}{\Gamma(1+5 \alpha)}\right) \\
& \times E_{\alpha}\left(-y^{\alpha}\right)+E_{\alpha}\left(-y^{\alpha}\right){ }_{0} I_{x}^{(\alpha)} \\
& \times \frac{(s-x)^{\alpha}}{\Gamma(1+\alpha)}\left(\frac{4 x^{5 \alpha}}{\Gamma(1+5 \alpha)}\right) \\
& =\frac{x^{\alpha}}{\Gamma(1+\alpha)} E_{\alpha}\left(-y^{\alpha}\right)-\frac{x^{3 \alpha}}{\Gamma(1+3 \alpha)} E_{\alpha}\left(-y^{\alpha}\right) \\
& +\frac{2 x^{5 \alpha}}{\Gamma(1+5 \alpha)} E_{\alpha}\left(-y^{\alpha}\right)-\frac{4 x^{7 \alpha}}{\Gamma(1+7 \alpha)} E_{\alpha}\left(-y^{\alpha}\right) \\
& =\left(\frac{x^{\alpha}}{\Gamma(1+\alpha)}-\frac{x^{3 \alpha}}{\Gamma(1+3 \alpha)}\right. \\
& \left.+\frac{2 x^{5 \alpha}}{\Gamma(1+5 \alpha)}-\frac{4 x^{7 \alpha}}{\Gamma(1+7 \alpha)}\right) E_{\alpha}\left(-y^{\alpha}\right) .
\end{aligned}
$$

From (16) and (20), we obtain the fourth approximation of (14) given as

$$
\begin{aligned}
M_{4}(x, y)= & M_{3}(x, y)+{ }_{0} I_{x}^{(\alpha)} \frac{(s-x)^{\alpha}}{\Gamma(1+\alpha)} \\
\times & \left\{\frac{\partial^{2 \alpha} M_{3}(x, y)}{\partial x^{2 \alpha}}+\frac{\partial^{2 \alpha} M_{3}(x, y)}{\partial y^{2 \alpha}}+M_{3}(x, y)\right. \\
& \left.-\frac{x^{\alpha}}{\Gamma(1+\alpha)} E_{\alpha}\left(-y^{\alpha}\right)\right\}
\end{aligned}
$$


4

Mathematical Problems in Engineering

$$
\begin{aligned}
= & \left(\frac{x^{\alpha}}{\Gamma(1+\alpha)}-\frac{x^{3 \alpha}}{\Gamma(1+3 \alpha)}+\frac{2 x^{5 \alpha}}{\Gamma(1+5 \alpha)}\right. \\
& \left.\quad-\frac{4 x^{7 \alpha}}{\Gamma(1+7 \alpha)}\right) E_{\alpha}\left(-y^{\alpha}\right)+E_{\alpha}\left(-y^{\alpha}\right)_{0} I_{x}^{(\alpha)} \\
& \times \frac{(s-x)^{\alpha}}{\Gamma(1+\alpha)}\left(-\frac{8 x^{7 \alpha}}{\Gamma(1+7 \alpha)}\right) \\
= & \frac{x^{\alpha}}{\Gamma(1+\alpha)} E_{\alpha}\left(-y^{\alpha}\right)-\frac{x^{3 \alpha}}{\Gamma(1+3 \alpha)} E_{\alpha}\left(-y^{\alpha}\right) \\
& +\frac{2 x^{5 \alpha}}{\Gamma(1+5 \alpha)} E_{\alpha}\left(-y^{\alpha}\right)-\frac{4 x^{7 \alpha}}{\Gamma(1+7 \alpha)} E_{\alpha}\left(-y^{\alpha}\right) \\
& +\frac{8 x^{9 \alpha}}{\Gamma(1+9 \alpha)} E_{\alpha}\left(-y^{\alpha}\right) \\
= & \left(\frac{4 x^{7 \alpha}}{\Gamma(1+7 \alpha)}+\frac{8 x^{9 \alpha}}{\Gamma(1+9 \alpha)}\right) E_{\alpha}\left(-y^{\alpha}\right) . \\
\Gamma(1+\alpha) & \frac{x^{3 \alpha}}{\Gamma(1+3 \alpha)}+\frac{2 x^{5 \alpha}}{\Gamma(1+5 \alpha)}
\end{aligned}
$$

As similar manner, from (21), we arrive at the fifth approximate formula:

$$
\begin{aligned}
& M_{5}(x, y)=M_{4}(x, y)+{ }_{0} I_{x}^{(\alpha)} \frac{(s-x)^{\alpha}}{\Gamma(1+\alpha)} \\
& \times\left\{\frac{\partial^{2 \alpha} M_{4}(x, y)}{\partial x^{2 \alpha}}+\frac{\partial^{2 \alpha} M_{4}(x, y)}{\partial y^{2 \alpha}}+M_{4}(x, y)\right. \\
& \left.-\frac{x^{\alpha}}{\Gamma(1+\alpha)} E_{\alpha}\left(-y^{\alpha}\right)\right\} \\
& =\left(\frac{x^{\alpha}}{\Gamma(1+\alpha)}-\frac{x^{3 \alpha}}{\Gamma(1+3 \alpha)}+\frac{2 x^{5 \alpha}}{\Gamma(1+5 \alpha)}\right. \\
& \left.-\frac{4 x^{7 \alpha}}{\Gamma(1+7 \alpha)}+\frac{8 x^{9 \alpha}}{\Gamma(1+9 \alpha)}\right) E_{\alpha}\left(-y^{\alpha}\right) \\
& +E_{\alpha}\left(-y^{\alpha}\right)_{0} I_{x}^{(\alpha)} \frac{(s-x)^{\alpha}}{\Gamma(1+\alpha)}\left(\frac{16 x^{9 \alpha}}{\Gamma(1+9 \alpha)}\right) \\
& =\frac{x^{\alpha}}{\Gamma(1+\alpha)} E_{\alpha}\left(-y^{\alpha}\right)-\frac{x^{3 \alpha}}{\Gamma(1+3 \alpha)} E_{\alpha}\left(-y^{\alpha}\right) \\
& +\frac{2 x^{5 \alpha}}{\Gamma(1+5 \alpha)} E_{\alpha}\left(-y^{\alpha}\right)-\frac{4 x^{7 \alpha}}{\Gamma(1+7 \alpha)} E_{\alpha}\left(-y^{\alpha}\right) \\
& +\frac{8 x^{9 \alpha}}{\Gamma(1+9 \alpha)} E_{\alpha}\left(-y^{\alpha}\right)-\frac{16 x^{11 \alpha}}{\Gamma(1+11 \alpha)}
\end{aligned}
$$

$$
\begin{aligned}
& =\left(\frac{x^{\alpha}}{\Gamma(1+\alpha)}-\frac{x^{3 \alpha}}{\Gamma(1+3 \alpha)}+\frac{2 x^{5 \alpha}}{\Gamma(1+5 \alpha)}\right. \\
& \left.\quad-\frac{4 x^{7 \alpha}}{\Gamma(1+7 \alpha)}+\frac{8 x^{9 \alpha}}{\Gamma(1+9 \alpha)}-\frac{16 x^{11 \alpha}}{\Gamma(1+11 \alpha)}\right) \\
& \quad \times E_{\alpha}\left(-y^{\alpha}\right) .
\end{aligned}
$$

Hence, we have the local fractional series solution of (14):

$$
\begin{aligned}
& M_{n}(x, y) \\
& =\left(\frac{x^{\alpha}}{\Gamma(1+\alpha)}-\frac{x^{3 \alpha}}{\Gamma(1+3 \alpha)}+\frac{2 x^{5 \alpha}}{\Gamma(1+5 \alpha)}\right. \\
& \left.\quad \quad-\frac{4 x^{7 \alpha}}{\Gamma(1+7 \alpha)}+\frac{8 x^{9 \alpha}}{\Gamma(1+9 \alpha)}-\frac{16 x^{11 \alpha}}{\Gamma(1+11 \alpha)}+\cdots\right) \\
& \quad \times E_{\alpha}\left(-y^{\alpha}\right) \\
& =\left(\frac{1}{2} \frac{x^{\alpha}}{\Gamma(1+\alpha)}+\frac{1}{2} \sum_{i=0}^{\infty}(-1)^{i} \frac{2^{i} x^{(2 i+1) \alpha}}{\Gamma(1+(2 i+1) \alpha)}\right) \\
& \quad \times E_{\alpha}\left(-y^{\alpha}\right) .
\end{aligned}
$$

From (13), we get the exact solution of (14) given as

$$
\begin{aligned}
M= & \lim _{n \rightarrow \infty} M_{n}(x, y) \\
= & \lim _{n \rightarrow \infty}\left(\frac{1}{2} \frac{x^{\alpha}}{\Gamma(1+\alpha)}+\frac{1}{2} \sum_{i=0}^{\infty}(-1)^{i} \frac{2^{i} x^{(2 i+1) \alpha}}{\Gamma(1+(2 i+1) \alpha)}\right) \\
& \times E_{\alpha}\left(-y^{\alpha}\right) \\
= & {\left[\frac{1}{2} \frac{x^{\alpha}}{\Gamma(1+\alpha)}+\frac{1}{2} \sin _{\alpha}\left(2 x^{\alpha}\right)\right] E_{\alpha}\left(-y^{\alpha}\right) }
\end{aligned}
$$

and its plot is illustrated in Figure 2.

Example 1. We suggest the following local fractional inhomogeneous Helmholtz equation:

$$
\begin{gathered}
\frac{\partial^{2 \alpha} M(x, y)}{\partial x^{2 \alpha}}+\frac{\partial^{2 \alpha} M(x, y)}{\partial y^{2 \alpha}}+M(x, y) \\
=\frac{x^{\alpha}}{\Gamma(1+\alpha)} \frac{y^{\alpha}}{\Gamma(1+\alpha)},
\end{gathered}
$$

and the initial-boundary conditions read as

$$
\begin{gathered}
\frac{\partial^{\alpha} M(0, y)}{\partial x^{\alpha}}=\frac{y^{\alpha}}{\Gamma(1+\alpha)}, \\
M(0, y)=0 .
\end{gathered}
$$




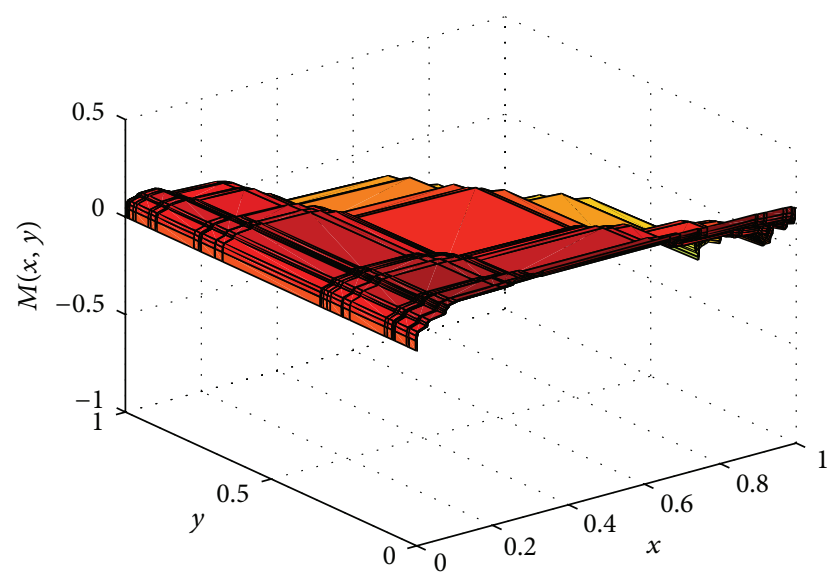

FIgURE 2: The graph of exact solution of (14) where $\alpha=\ln 2 / \ln 3$.

From (11), we set up the local fractional variational iteration algorithm as follows:

$$
\begin{aligned}
& M_{n+1}(x, y) \\
& =M_{n}(x, y)+{ }_{0} I_{x}^{(\alpha)} \frac{(s-x)^{\alpha}}{\Gamma(1+\alpha)} \\
& \quad \times\left\{\frac{\partial^{2 \alpha} M_{n}(x, y)}{\partial x^{2 \alpha}}+\frac{\partial^{2 \alpha} M_{n}(x, y)}{\partial y^{2 \alpha}}\right. \\
& \left.\quad+M_{n}(x, y)-\frac{x^{\alpha}}{\Gamma(1+\alpha)} \frac{y^{\alpha}}{\Gamma(1+\alpha)}\right\},
\end{aligned}
$$

where the initial value is suggested as

$$
M_{0}(x, y)=\frac{x^{\alpha}}{\Gamma(1+\alpha)} \frac{y^{\alpha}}{\Gamma(1+\alpha)}
$$

Appling (27) and (28) gives the first approximate solution:

$$
\begin{aligned}
M_{1}(x, y) & \\
= & M_{0}(x, y)+{ }_{0} I_{x}^{(\alpha)} \frac{(s-x)^{\alpha}}{\Gamma(1+\alpha)} \\
& \times\left\{\frac{\partial^{2 \alpha} M_{0}(x, y)}{\partial x^{2 \alpha}}+\frac{\partial^{2 \alpha} M_{0}(x, y)}{\partial y^{2 \alpha}}+M_{0}(x, y)\right. \\
& \left.-\frac{x^{\alpha}}{\Gamma(1+\alpha)} \frac{y^{\alpha}}{\Gamma(1+\alpha)}\right\} \\
= & \frac{x^{\alpha}}{\Gamma(1+\alpha)} \frac{y^{\alpha}}{\Gamma(1+\alpha)} .
\end{aligned}
$$

Using (27) and (29), we obtain the second approximate term, which is expressed as follows:

$$
\begin{aligned}
M_{2}(x, y) & \\
= & M_{1}(x, y)+{ }_{0} I_{x}^{(\alpha)} \frac{(s-x)^{\alpha}}{\Gamma(1+\alpha)} \\
& \times\left\{\frac{\partial^{2 \alpha} M_{1}(x, y)}{\partial x^{2 \alpha}}+\frac{\partial^{2 \alpha} M_{1}(x, y)}{\partial y^{2 \alpha}}+M_{1}(x, y)\right. \\
& \left.-\frac{x^{\alpha}}{\Gamma(1+\alpha)} \frac{y^{\alpha}}{\Gamma(1+\alpha)}\right\} \\
= & \frac{x^{\alpha}}{\Gamma(1+\alpha)} \frac{y^{\alpha}}{\Gamma(1+\alpha)} .
\end{aligned}
$$

In view of (27) and (30), we obtain the third approximation, which reads as follows:

$$
\begin{aligned}
M_{3}(x, y) & \\
= & M_{2}(x, y)+{ }_{0} I_{x}^{(\alpha)} \frac{(s-x)^{\alpha}}{\Gamma(1+\alpha)} \\
& \times\left\{\frac{\partial^{2 \alpha} M_{2}(x, y)}{\partial x^{2 \alpha}}+\frac{\partial^{2 \alpha} M_{2}(x, y)}{\partial y^{2 \alpha}}+M_{2}(x, y)\right. \\
& \left.\quad-\frac{x^{\alpha}}{\Gamma(1+\alpha)} \frac{y^{\alpha}}{\Gamma(1+\alpha)}\right\} \\
= & \frac{x^{\alpha}}{\Gamma(1+\alpha)} \frac{y^{\alpha}}{\Gamma(1+\alpha)} .
\end{aligned}
$$

Therefore, we arrive at the approximate term

$$
M_{n}(x, y)=\frac{x^{\alpha}}{\Gamma(1+\alpha)} \frac{y^{\alpha}}{\Gamma(1+\alpha)},
$$

which leads to the exact solution of (25) given as

$$
\begin{aligned}
M & (x, y) \\
& =\lim _{n \rightarrow \infty} M_{n}(x, y) \\
& =\lim _{n \rightarrow \infty} \frac{x^{\alpha}}{\Gamma(1+\alpha)} \frac{y^{\alpha}}{\Gamma(1+\alpha)} \\
& =\frac{x^{\alpha}}{\Gamma(1+\alpha)} \frac{y^{\alpha}}{\Gamma(1+\alpha)}
\end{aligned}
$$

and its plot is illustrated in Figure 3.

\section{Conclusions}

In this work, the boundary value problems for the inhomogeneous Helmholtz equation within local fractional derivative operator were discussed by using the local fractional variational iteration method. Their nondifferentiable solutions are obtained and the graphs of the solutions with fractal dimension $\alpha=\ln 2 / \ln 3$ are also given. 


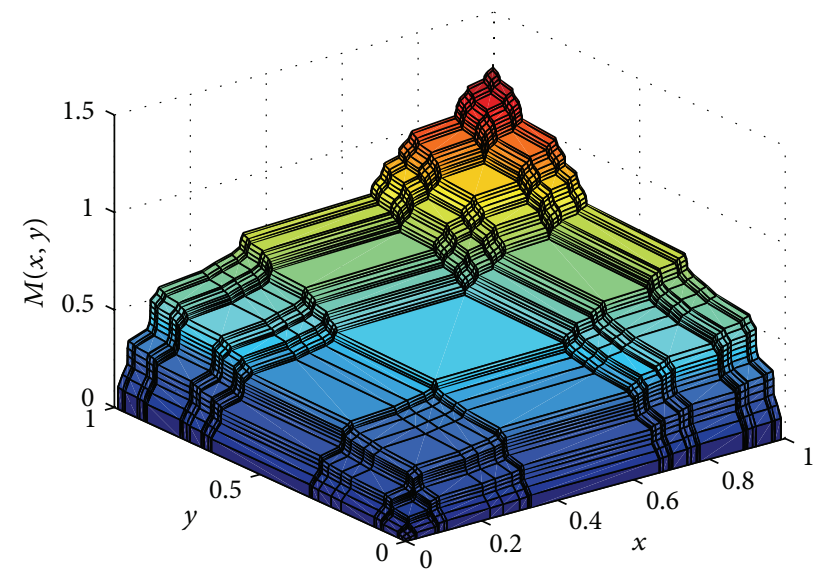

Figure 3: The plot of exact solution of (25) where $\alpha=\ln 2 / \ln 3$.

\section{Conflict of Interests}

The authors declare that they have no competing interests in this paper.

\section{Acknowledgment}

This work was supported by the Zhejiang Provincial Natural Science Foundation (LY13A010007).

\section{References}

[1] A. Sommerfeld, Partial Differential Equations in Physics, Academic Press, New York, NY, USA, 1949.

[2] K. F. Riley, M. P. Hobson, and S. J. Bence, Mathematical Methods for Physics and Engineering, Cambridge University Press, Cambridge, UK, 2006.

[3] F. Ihlenburg and I. Babuška, "Finite element solution of the Helmholtz equation with high wave number Part I: the hversion of the FEM," Computers and Mathematics with Applications, vol. 30, no. 9, pp. 9-37, 1995.

[4] S. Momani and S. Abuasad, "Application of He's variational iteration method to Helmholtz equation," Chaos, Solitons \& Fractals, vol. 27, no. 5, pp. 1119-1123, 2006.

[5] M. Rafei and D. D. Ganji, "Explicit solutions of Helmholtz equation and fifth-order KdV equation using homotopy perturbation method," International Journal of Nonlinear Sciences and Numerical Simulation, vol. 7, no. 3, pp. 321-328, 2006.

[6] A. Bayliss, C. I. Goldstein, and E. Turkel, "An iterative method for the Helmholtz equation," Journal of Computational Physics, vol. 49, no. 3, pp. 443-457, 1983.

[7] J. Benamou and B. Desprès, "A domain decomposition method for the Helmholtz equation and related optimal control problems," Journal of Computational Physics, vol. 136, no. 1, pp. 6882, 1997.

[8] C. M. Linton, “The Green's function for the two-dimensional Helmholtz equation in periodic domains," Journal of Engineering Mathematics, vol. 33, no. 4, pp. 377-402, 1998.

[9] I. Singer and E. Turkel, "High-order finite difference methods for the Helmholtz equation," Computer Methods in Applied Mechanics and Engineering, vol. 163, no. 1-4, pp. 343-358, 1998.
[10] K. Otto and E. Larsson, "Iterative solution of the Helmholtz equation by a second-order method," SIAM Journal on Matrix Analysis and Applications, vol. 21, no. 1, pp. 209-229, 1999.

[11] A. A. Kilbas, H. M. Srivastava, and J. J. Trujillo, Theory and Applications of Fractional Differential Equations, New York, NY, USA, Elsevier, 2006.

[12] C. Li, Y. Chen, and J. Kurths, "Fractional calculus and its applications," Philosophical Transactions of the Royal Society of London A: Mathematical, Physical and Engineering Sciences, vol. 371, no. 1990, Article ID 20130037, 3 pages, 2013.

[13] J. A. T. Machado, "Analysis and design of fractional-order digital control systems," Systems Analysis Modelling Simulation, vol. 27, no. 2-3, pp. 107-122, 1997.

[14] R. C. Koeller, "Applications of fractional calculus to the theory of viscoelasticity," Journal of Applied Mechanics, vol. 51, no. 2, pp. 299-307, 1984.

[15] V. Namias, "The fractional order Fourier transform and its application to quantum mechanics," Journal of Applied Mathematics, vol. 25, no. 3, pp. 241-265, 1980.

[16] R. Metzler and J. Klafter, "The random walk's guide to anomalous diffusion: a fractional dynamics approach," Physics Reports, vol. 339, no. 1, pp. 1-77, 2000.

[17] M. S. Samuel and A. Thomas, "On fractional Helmholtz equations," Fractional Calculus \& Applied Analysis, vol. 13, no. 3, pp. 295-308, 2010.

[18] A. Atangana and S. B. Belhaouari, "Solving partial differential equation with space- and time-fractional derivatives via homotopy decomposition method," Mathematical Problems in Engineering, vol. 2013, Article ID 318590, 9 pages, 2013.

[19] A. Atangana and A. Kilicman, "A novel integral operator transform and its application to some FODE and FPDE with some kind of singularities," Mathematical Problems in Engineering, vol. 2013, Article ID 531984, 7 pages, 2013.

[20] A. H. Bhrawy, A. S. Alofi, and S. S. Ezz-Eldien, "A quadrature tau method for fractional differential equations with variable coefficients," Applied Mathematics Letters, vol. 24, no. 12, pp. 2146-2152, 2011.

[21] E. H. Doha, A. H. Bhrawy, and S. S. Ezz-Eldien, "A new Jacobi operational matrix: an application for solving fractional differential equations," Applied Mathematical Modelling, vol. 36, no. 10, pp. 4931-4943, 2012.

[22] V. Daftardar-Gejji and H. Jafari, "Adomian decomposition: a tool for solving a system of fractional differential equations," Journal of Mathematical Analysis and Applications, vol. 301, no. 2, pp. 508-518, 2005.

[23] H. Jafari and S. Seifi, "Homotopy analysis method for solving linear and nonlinear fractional diffusion-wave equation," Communications in Nonlinear Science and Numerical Simulation, vol. 14, no. 5, pp. 2006-2012, 2009.

[24] X.-J. Yang, Advanced Local Fractional Calculus and Its Applications, World Science, New York, NY, USA, 2012.

[25] X. J. Yang, D. Baleanu, and J. A. T. Machado, "Mathematical aspects of the Heisenberg uncertainty principle within local fractional Fourier analysis," Boundary Value Problems, vol. 2013, article 131, 2013.

[26] X. Yang, H. M. Srivastava, J. H. He, and D. Baleanu, "Cantortype cylindrical-coordinate method for differential equations with local fractional derivatives," Physics Letters A, vol. 377, no. 28-30, pp. 1696-1700, 2013.

[27] W. Zhong, F. Gao, and X. Shen, "Applications of Yang-Fourier transform to local fractional equations with local fractional 
derivative and local fractional integral," Advanced Materials Research, vol. 461, pp. 306-310, 2012.

[28] C. Long, Y. Zhao, and H. Jafari, "Mathematical models arising in the fractal forest gap via local fractional calculus," Abstract and Applied Analysis, vol. 2014, Article ID 782393, 6 pages, 2014.

[29] Y. Hao, H. M. Srivastava, H. Jafari, and X.-J. Yang, "Helmholtz and diffusion equations associated with local fractional derivative operators involving the Cantorian and Cantor-type cylindrical coordinates," Advances in Mathematical Physics, vol. 2013, Article ID 754248, 5 pages, 2013.

[30] A. M. Yang, Z. S. Chen, H. M. Srivastava, and X. J. Yang, "Application of the local fractional series expansion method and the variational iteration method to the Helmholtz equation involving local fractional derivative operators," Abstract and Applied Analysis, vol. 2013, Article ID 259125, 6 pages, 2013.

[31] X.-J. Yang and D. Baleanu, "Fractal heat conduction problem solved by local fractional variation iteration method," Thermal Science, vol. 17, no. 2, pp. 625-628, 2013.

[32] W. Su, D. Baleanu, X. J. Yang, and H. Jafari, "Damped wave equation and dissipative wave equation in fractal strings within the local fractional variational iteration method," Fixed Point Theory and Applications, vol. 2013, article 89, 2013.

[33] D. Baleanu, J. A. T. Machado, C. Cattani, M. C. Baleanu, and $\mathrm{X}$. Yang, "Local fractional variational iteration and decomposition methods for wave equation on Cantor sets within local fractional operators," Abstract and Applied Analysis, vol. 2014, Article ID 535048, 6 pages, 2014.

[34] J.-H. He, "Local fractional variational iteration method for fractal heat transfer in silk cocoon hierarchy," Nonlinear Science Letters A, vol. 4, no. 1, pp. 15-20, 2013. 


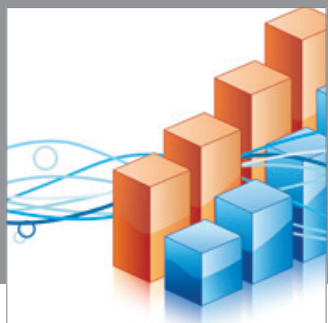

Advances in

Operations Research

mansans

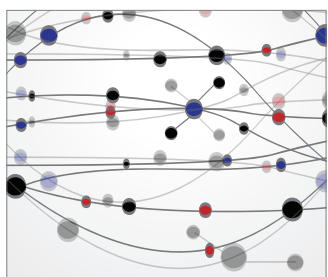

The Scientific World Journal
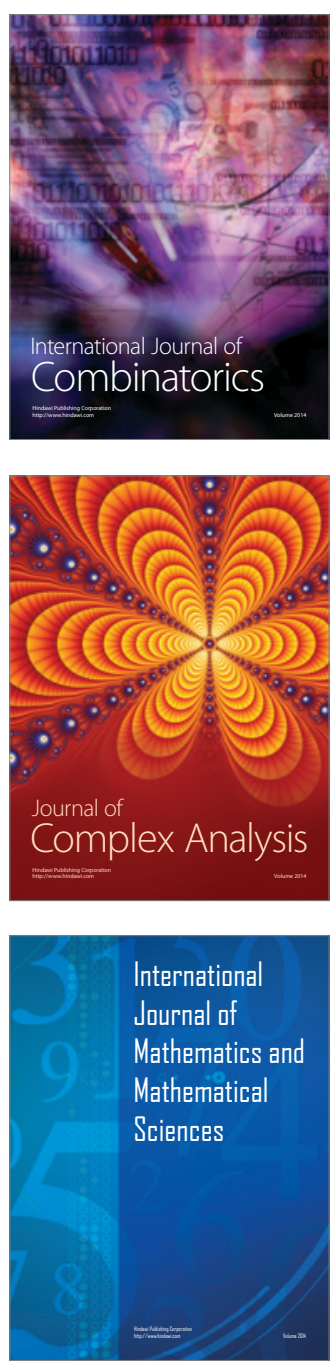
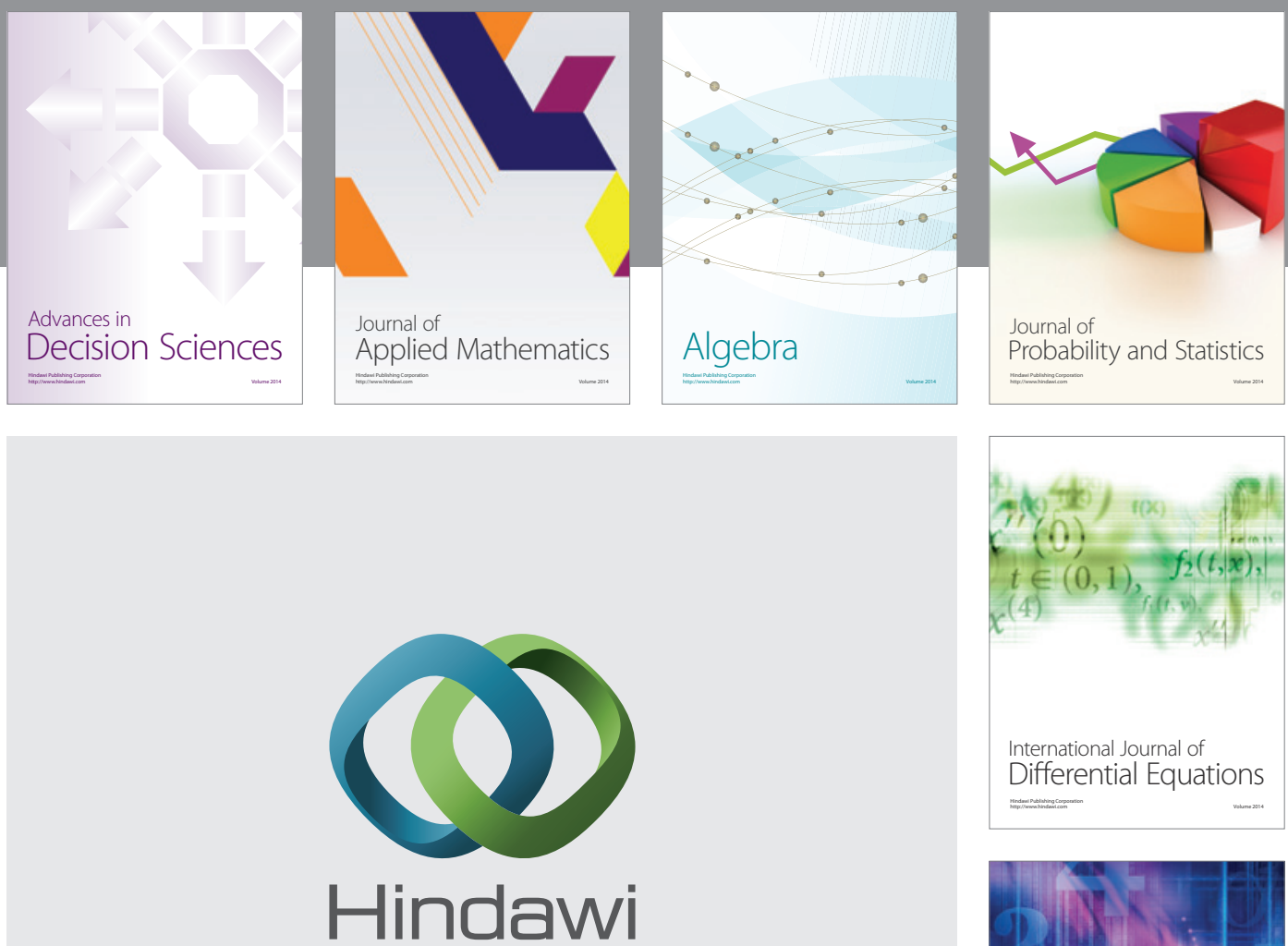

Submit your manuscripts at http://www.hindawi.com
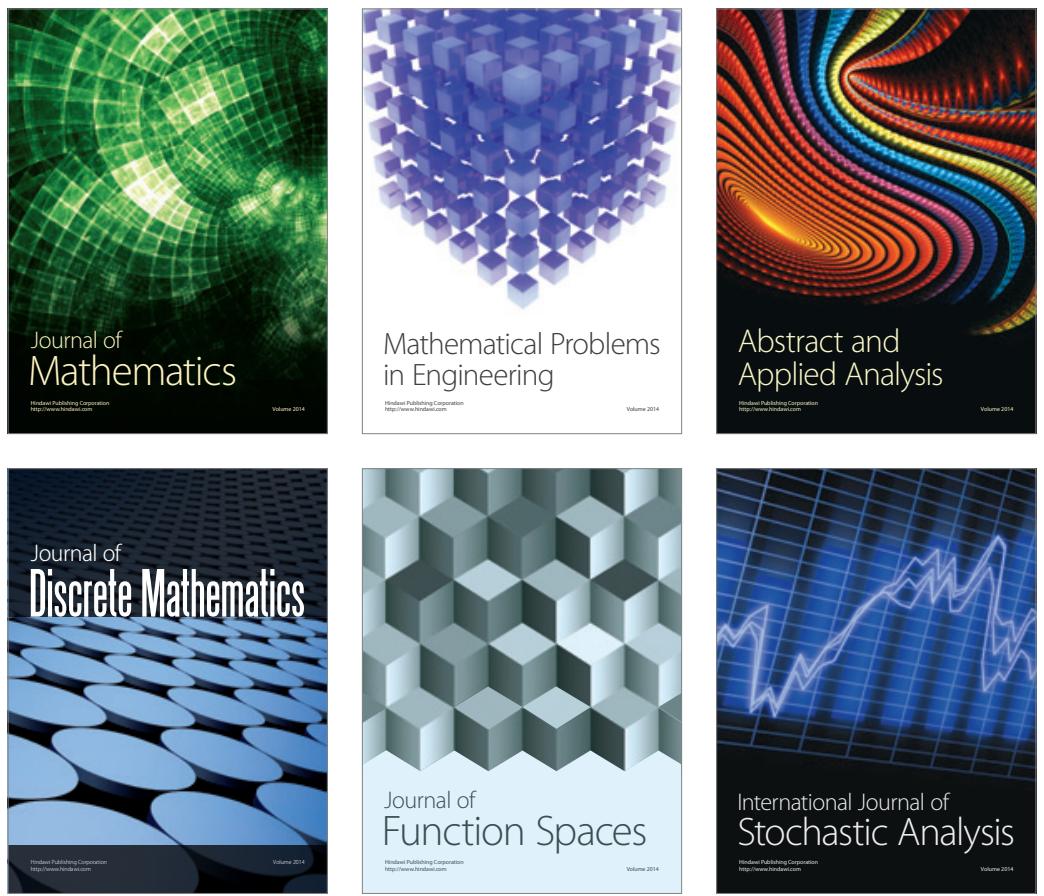

Journal of

Function Spaces

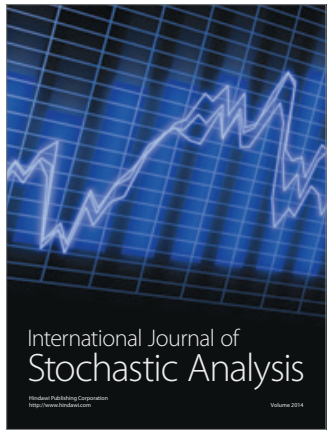

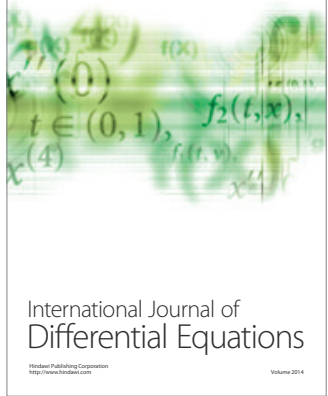
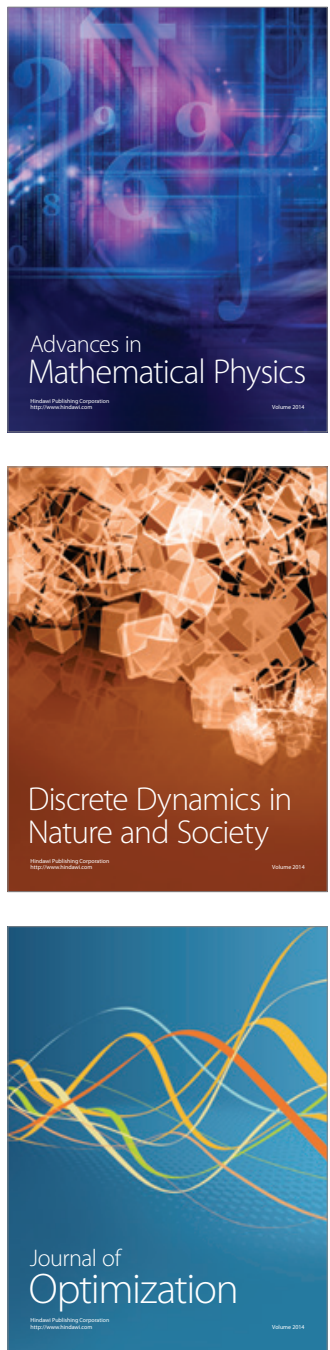